\title{
Optimal Route Search in Mobile Ad-Hoc Network Using Ant Colony Optimization with Power Saving Technique
}

\author{
Bandana Mahapatra \\ Department of Computer Sc. \& Engg. Institute of Technical Education \& Research, \\ Siksha 'O' Anusandhan University, Bhubaneswar.
}

\begin{abstract}
In Ad-Hoc networks the mobile nodes communicate with each other using multi-hop wireless links. The main drawback of such network is that there are no stationary infrastructures to route the packets. Hence, routing protocols have to adapt quickly and elegantly to frequent and unpredictable changes in network technology and they have to do so while conserving the memory, power and bandwidth resource. The Ant Colony Optimization technique implemented upon such networks have helped the nodes in finding the routes to different nodes in an optimized way same as ants find the optimum route to its food. The techniques provided so far have considered the search space utilized by a node as the space occupied by all the nodes that are present in the network which requires message passing among all the nodes that are present in the network consuming plenty of bandwidth and power only to find the routes to different nodes. If we divide the search space among the nodes forming clusters then the number of messages communicating will be reduced thereby reducing the bandwidth occupied and power consumed conserving the important resources of mobile communication in wireless medium.
\end{abstract}

Key words: Mobile Ad-hoc Networks, Optimal Route Searching ,Ant Colony Optimization.

\section{Introduction}

Mobile Ad-hoc Networks have grown in popularity over the recent advancements in technology as they possess the ability to provide instant wireless networking solution in places/situations where no pre-deployed infrastructure exists. MANETs prove to be an attractive solution in a wide of environments ranging from scattered military deployment to simple network of a group of notebooks in an office meeting. In all cases, there is a need for a group of nodes (laptops, desktops, pdas, cell phones, etc) to group together and create a network which can offer services like file-sharing, messaging, resource sharing, etc. Hence the primary goal in a mobile network is to efficiently establish one or more routes between two nodes so that they can communicate reliably. We are all familiar with swarms in nature. The word swarm conjures up images of large groups of small insects in which each member performs a simple role, but the action produces complex behavior as a whole. The emergence of such complex behavior extends beyond swarms. Similar complex social structures also occur in higher-order animals and insects that don't swarm: colonies of ants, flocks of birds, or packs of wolves.

These groups behave like swarms in many ways. Wolves, for example, accept the alpha male and female as leaders that communicate with the pack via body language and facial expressions. The alpha male marks his pack's territory and excludes wolves that are not members.

Several areas of computer science have adopted the idea that swarms can solve complex problems. For our purposes, the term swarm refers to a large group of simple components working together to achieve a goal and produce significant results. Swarms may operate on or under the Earth's surface, under water, or on other planets.

Optimization Algorithms are becoming increasingly popular in engineering design activities, primarily because of the availability of affordability of high speed computers. They are extensively used in those engineering design problems where the emphasis is on maximizing or minimizing a certain goal. For example, optimization algorithms are routinely used in aerospace design activities to minimize the overall weight simply because every element or component adds to the overall weight of the aircraft. Thus optimization is a term used for either minimization or maximization of an objective.

As we know the mobile ad-hoc networks consist of the nodes that are required to perform the functions without any proper infrastructure with limited resources that is battery power and bandwidth. This paper proposes an algorithm to find out the optimal route from each of the nodes to other nodes along with conserving these resources. 


\section{Ad- Hoc Networks}

In an Ad-Hoc network, each node works as a host as well as a router. This needs each node to maintain some form of information regarding the network around it along with some algorithm governing the sending and receiving of data packets. This Algorithm together with the supporting information regarding network condition is called a routing protocol

In an Ad-Hoc network, each node works as a host as well as a router. This needs each node to maintain some form of information regarding the network around it along with some algorithm governing the sending and receiving of data packets. This Algorithm together with the supporting information regarding network condition is called a routing protocol Routing Protocol in ad hoc network has to adapt quickly and elegantly to frequent unpredictable changes in network topology, and they have to do so while conserving memory power and bandwidth resources. When ad-hoc networks are scaled up, they generally encounter excessive overhead in routing messages, caused due to growing number of nodes and amplified by higher node mobility. Growing Networks also lead to excessive routing table sizes, which typically have to be broadcasted to other nodes again adding to network overhead.

In an Ad-Hoc network, each node works as a host as well as a router. This needs each node to maintain some form of information regarding the network around it along with some algorithm governing the sending and receiving of data packets. This Algorithm together with the supporting information regarding network condition is called a routing protocol Routing Protocol in ad hoc network has to adapt quickly and elegantly to frequent unpredictable changes in network topology, and they have to do so while conserving memory power and bandwidth resources. When ad-hoc networks are scaled up, they generally encounter excessive overhead in routing messages, caused due to growing number of nodes and amplified by higher node mobility. Growing Networks also lead to excessive routing table sizes, which typically have to be broadcasted to other nodes again adding to network overhead.

2.1. Classification of Ad Hoc Protocols : The above Figure 1 presents a Hierarchical overview of ad-hoc routing protocols. It has to be noted that there are many different axis along which to classify ad-hoc routing protocols. Lang maintains that a classification along the hierarchical or flat networks structures is not valid since a flat network structure is not really a property in itself, but rather the absence of hierarchical property"Ref.[1]". However since some networks generally lend themselves better to hierarchical networks and vice-versa, a network designer may find a classification along the hierarchical or flat network structure axis on basis that it yields useful information, even though such classification can be argued to be logically incorrect.

Ad-hoc routing protocols

\begin{tabular}{llllll} 
Hierarchical protocols & \multicolumn{3}{c}{ Flat protocols } \\
Proactive & Reactive & Hybrid & Proactive & Reactive & Hybrid \\
STAR & CERP & ZHLS & FSR & AODV & ZRP \\
MMWN & & SLURP & FSLS & DSR & \\
CGSR & & DST & LSR & ROAM & \\
HSR & & DDR & TBRPF & LMR &
\end{tabular}

\section{Figure 1: TAXONOMY OF AD-HOC ROUTING PROTOCOLS}

\subsubsection{Flat Routing Protocols}

In flat routing protocol all hosts are treated equally and therefore host can be used to forward packets between arbitrary sources and destinations. In general a set of homogenous processes is applied at each host. These processes include information collection, mobility management and routing.

\subsubsection{Hierarchical Routing Protocol}

To permit scaling hierarchical techniques are usually applied. The major advantage of hierarchical routing is the reduction of routing table storage and processing overhead "Ref.[2][3]". Among hierarchical routing scheme the cluster based algorithm divides the given graph into a number of non-redundant clusters that may overlap with each other. Each node within the same cluster can communicate with each other directly by sending message but communicates to another node in another cluster through cluster head.

\section{Ant Colony Optimization}

Less than a hundred years ago the building of termite mounds, the nest building of the social wasps and ability of ants to converge on source food were considered somewhat magical aspect of nature. How could these simple, seemingly uncommunicative creatures be responsible for such epic feats of construction and 
organization? Biologists have over the last century unraveled many of these mystery and provided the foundation for fields of research variously known as collective intelligence, swarm intelligence and emergent behavior.

Less than a hundred years ago the building of termite mounds, the nest building of the social wasps and ability of ants to converge on source food were considered somewhat magical aspect of nature. How could these simple, seemingly uncommunicative creatures be responsible for such epic feats of construction and organization? Biologists have over the last century unraveled many of these mystery and provided the foundation for fields of research variously known as collective intelligence, swarm intelligence and emergent behavior.

\subsection{Stigmergy and Self -Organization}

In 1959, Grasse published the result of a study of termites in which he noted that the termites tended to follow a set of very simple rules when constructing there nest "Ref.[4]"..

- First, the termites move around random, dropping pallets of chewed earth and saliva on any slightly elevated patch of ground they encounter. This soon cause small heaps of moist earth to form.

- These heaps of salivated earth encouraged other termites to drop more pallets in the same place. Soon the biggest heaps start to develop in columns, which will continue to be built until a certain height is reached.

- If the columns were built close enough to other columns, the termites will start climbing each column and start building diagonally towards the neighboring columns.

Stigmergy, therefore is a mechanism which enables an environment to structure itself through the activities of the agents in the environment

\subsection{Swarm Intelligence and the Ant Colony}

Ants have always been the fascinating subject for human beings. Several scientific books "Ref.[5][6]". and pure literature books "Ref.[7]" on ants have met with surprising public success. Individually they are remarkably simple creatures with limited memory and behavior that sometime seem to have random component. But collectively, ants consistently achieve remarkable feats of cooperation, coordination and construction. Document examples of these achievements include:

1. Emigration of a colony

2. Formation of chain bridges

3. Building of ant nests

4. Preferentially exploiting the richest available food source

3.3. The AntNet Routing Algorithm: The AntNet routing algorithm is a direct extension of simple ant colony optimization algorithm. Ant net is even closer to the real ants behavior that inspired the development of the ACO met heuristic than the ACO algorithms.

The ant net algorithm and its main characteristics can be summarized as follows.

-At regular intervals and concurrently with the data traffic from each network node artificial ants are asynchronously launched towards destination nodes selected according to the traffic distribution.

-Artificial ants act concurrently and independently and communicate in an indirect way through the pheromones they read and write locally on the nodes.

-Each artificial ant searches for minimum cost path joining its source and destination.

-Each artificial ant move step by step towards its destination node. At each intermediate node a greedy stochastic policy is applied to choose the next node to move to. The policy makes use of

1 .Node artificial pheromone

2. Node-local-artificial pheromone

3. Node-local problem dependent heuristic information

4. The ant's memory.

-While moving the artificial ants collect information about the time length, the congestion status, and the node identifiers of the following path.

-Once they are arrived at the destination the artificial ants go back to there source nodes by moving along the same path as before but in opposite direction.

- During this backward travel node local model of the network status and the pheromones stored at on each visited node are modified by the artificial ants as the function of the path they followed and of its goodness.

- Once they have returned to there source node the artificial ants are deleted from the system. 


\section{Optimization}

Optimization Algorithms are becoming increasingly popular in engineering design activities, primarily because of the availability of affordability of high-speed computers. They are extensively used in those engineering design problems where the emphasis is on maximizing or minimizing a certain goal. For example, optimization algorithms are routinely used in aerospace design activities to minimize the overall weight simply because every element or component adds to the overall weight of the aircraft.

Thus optimization is a term used for either minimization or maximization of an objective.

\subsection{Optimization Algorithms}

The above optimization problems reveal the fact that the formulation of engineering design problems could differ from problem to problem. Certain problems involve linear terms for constraints and objective function and certain other problems involve non linear terms for them. In some problems, the terms are not explicit function of the design variables. Unfortunately there does not exist a single optimization algorithm, which will work in all optimization problems equally efficiently. Some algorithms perform better on one problem but may perform poorly on other problems. That is why the optimization literature contains a large number of algorithms each suitable to solve a particular type of problem. The choice of algorithm for an optimization problem is, to a large extent, dependent on the users experience in solving similar problems.

\subsubsection{Single variable optimization algorithms}

Because of there simplicity, single variable optimization techniques are discussed first. These algorithms provide a good understanding of the properties of the minimum and maximum points in a function and how optimization algorithms work iteratively to find the optimum point in a problem. The algorithms are classified into two categories- direct methods and gradient based methods. Direct methods do not use any derivative information of the objective function; only objective function values are used to guide the search process. However, gradient based methods use derivative information (first and/or second order) to guide search process. Although engineering optimization problems usually contain more than one design variables, single variable optimization algorithms are mainly used as unidirectional search methods in multivariable optimization algorithms.

\subsubsection{Multi-variable optimization algorithms}

A number of algorithms for unconstrained, multivariable optimization problems are discussed next. These algorithms demonstrate how the search for the optimum point progresses in multiple dimensions. Depending on weather the gradient information is used or not used, these algorithms are also classified into direct and gradient based techniques

\subsubsection{Constrained optimization algorithms}

Constrained optimization algorithms are described next. These algorithms use the single variable and multivariable optimization algorithms repeatedly and simultaneously maintain the search effort inside the feasible search region.

\subsubsection{Specialized optimization algorithms}

There exist a number of structured algorithms, which are ideal for only a certain class of optimization problems. Two of these algorithms- integer programming and geometric programming are often used in engineering design problems. Integer programming methods can solve optimization problems with integer design variables. Geometric programming methods solve optimization problems with objective functions and constraints written in a special form.

\subsubsection{Non traditional optimization algorithms}

There exist a number of other search optimization algorithms which are comparatively new and are becoming popular in engineering design optimization problems in the recent past. Two such algorithms are

- Genetic Algorithms.

- Simulated Annealing.

\section{Optimal Route Discovery in Mobile Ad hoc network Using Ant Colony Optimization}

The ant colony Optimization when implemented on the routing protocols replaced traditional tables with the pheromone tables where each node stores a routing table $T_{k}$ which is organized as in distance vector routing algorithm but stores probabilistic values for each of the destination pair $(\mathrm{d}, \mathrm{n}) . \mathrm{T}_{\mathrm{k}}$ stores a probabilistic value $\mathrm{P}_{\mathrm{nd}}$ which expresses the desirability of choosing $\mathrm{n}$ as the next hop node to destination such that : 


$$
\sum P_{n d}=1, d \in[1, \underset{n \in N K}{N}] \underset{k}{N_{k}}=\{\operatorname{neighbors}(k)\}
$$

A

B

\begin{tabular}{|ll|l|}
\hline $\mathrm{A}$ & & \\
& 0.35 & 0.57 \\
\hline $\mathrm{B}$ & & \\
& 0.76 & 0.88 \\
\hline
\end{tabular}

Example of an Ant Net Routing Tables

The above table for probability is calculated using the equation:

$$
\frac{\mathbf{P}_{\text {nd }}=\left(\mathbf{P}_{\text {nd }}+\mathbf{K}\right)^{\mathbf{F}}}{\sum_{\mathrm{i} \in \mathbf{N K}(\mathrm{t})}\left(\mathbf{P}_{\mathrm{id}}+\mathbf{K}\right)^{\mathbf{F}}}
$$

The metric for calculating the probability depends upon factors

$\mathrm{N}_{\mathrm{K}}(\mathrm{t})=$ set of known neighbors of node $\mathrm{K}$ at time $\mathrm{t}$.

$\mathrm{P}_{\mathrm{nd}} \quad=$ probability that a packet with destination $\mathrm{d}$ will choose node $\mathrm{n}$ as a next hop destination node $\mathrm{d}$

$\mathrm{P}_{\mathrm{nd}}=$ the amount of pheromone associated with a transition from the current node to node $\mathrm{n}$ for a packet with destination node $\mathrm{d}$.

$\mathrm{K}=\mathrm{A}$ constant that determines the sensitivity of the probability. Calculations to a small amount of pheromone i.e. a pheromone level offset.

$\mathrm{F}=\mathrm{A}$ constant that accentuates the difference between pheromone levels between links. $\mathrm{F}>1$ accentuates the difference in pheromone between links, and F,1 de-emphasize the difference.

The above value shows that the probability increases in the $P_{n d}, K$, and decreases with the factors like $P_{\text {id }}, \&$ $\mathrm{N}_{\mathrm{k}}(\mathrm{t})$.

There are many protocols developed based upon the Ant Colony Optimization technique for both the flat as well as Hierarchical Routing Protocol such as -

- Ant net

- Ant Colony Based Routing Algorithm

- Ant Hoc Net

- Ant AODV

- Termite

for Flat Routing Algorithm and

- $\quad$ Mobile Ants Based Routing (MABR)

- Adaptive SDR for hierarchical routing protocols.

These routing algorithms are diverse from each other in much respect. However many algorithms employ common sub mechanisms in the routing process.

1. Almost all algorithms employ common sub mechanism in routing process

2. Almost all algorithms use a variation of route request or forward packets to find destinations, with route reply or backward ant packets to find destinations, with route reply or backward ant packets completing the route.

3. Almost all algorithms send some forms of a route error packet to notify other nodes of link failures.

4. When a node is unable to deliver a packet to a next hop node, many algorithm employ some form of backtracking where the packet is returned to an upstream node in the hope that node can successfully forward the packet.

The general metric available to evaluate the protocols comes under the two classes

- Scenario metrics - it describes the environment in which the ad-hoc network operates. Thus the scenario metrics are not measures of the protocols performance but are the necessary parameters to compare the performance of protocols in different environment.

- Performance metric - describes the actual performance of the protocol, given a set of scenario metrics. The algorithm thesis proposes focuses mainly at improving upon following metric.

- Routing overhead ratio 
- Average power extended

- Route optimality.

\section{Which comes under the performance metrics?}

1. Routing overhead ratio - A network's byte delivery ratio is defined as the ratio of bytes originated by all nodes to data bytes received as final destination by all nodes in network excluding data bytes that originated outside the network boundary.

2. Average battery power expended - a nodes average power expended is defined as the average power spent by the node's per time period on the network related activities.

3. route optimality - a network's route optimality is a measure of the optimality of routes used by the network. In traditional fixed-lines networks. This is usually the ratio of the shortest available route to the route actually taken by any data packet. However in ad-hoc network factors such as power consumption and congestion avoidance have to be taken into consideration in addition to route length. Since this is the multi objective optimization problem, determination of the optimal route requires a measure of the Route's Perito Optimality.

Route Discovery in Ad- Hoc Network -

5.1.2. Route Discovery of DSR - Dynamic Source Routing (DSR) [4] is derived from the concept of source routing if a source needs a route to the destination node. It broadcasts a node request (ROUTE_REQ) packet to its neighbors. On a node receiving this request, two things may happen. If the node does not know a route to the destination, it appends its own address to the packet and propagates the route request packet to its neighbor. Thus, ROUTE_REQ packets can track paths leading to the destination. Loops can also be avoided by looking at the packet content. When the destination receives a ROUTE_REQ. it returns to a source node a route reply packet containing a route indicated in a ROUTE_REQ. The ROUTE_REPLY then travels, through unicast, in the reverse direction of the discovered route or on a path already known by the destination, to the source. The Source node, on receiving the ROUTE_REPLY, will place the route in its route cache.

In the second case, an intermediate node is also allowed to return a ROUTE_REPLY if is already known to a route fresh enough in its route cache. If so it simply concatenates the route in ROUTE_REQ and that in its route cache, and supplies this new route to the source. Also note that an intermediate node should register the ROUTE_REQ it has received to discard duplicate.

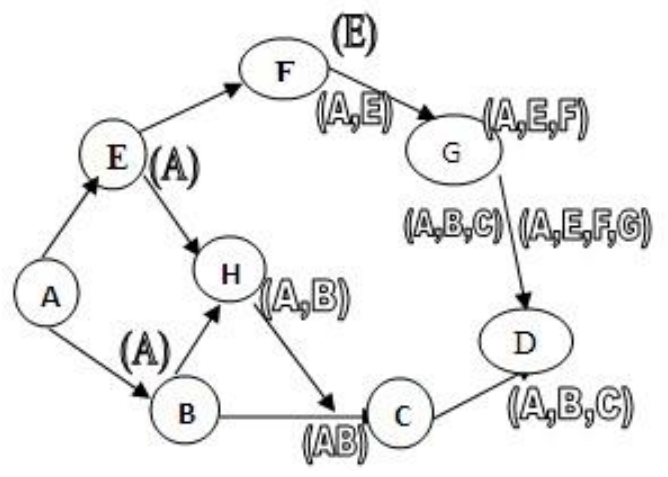

Fig 5.1.2 An example of Route Discovery in DSR, with A as the source and D as Destination.

The figure above shows the propagation of ROUTE_REQ packets. An arrow represents the transmission direction corresponding sender to receiver. The sequence of letters associated with each arrow indicates the traversed hosts that are recorded in the packet header.

5.1.3. Route Discovery of SSA - The signal stability adaptive protocol (SSA)[9] tries to discover longer lived routes based on signal strength and location stability. Each link is differentiated as strong or weak according to the average signal strength at which packets are heard. Beacons are sent periodically by each hosts for its neighbors to measure its stability. The protocol tends to choose the path that has existed for a long period of time. Each host maintains a signal stability table.

Like DSR, the SSA protocols also broadcasts ROUTE_REQUEST packets to discover routes. The source can also specify the quality of the route it desires. Possible route qualities are STRONG_LINK_ONLY, STRONG_PREFERRED, and NO_PREFERENCE. It is suggested that the STRONG_LINK_ONLY option be used in the first attempt. A receiving node should help propagating the request if:

1. the ROUTE_REQUEST is received over a strong link 
2. the REQUEST has not been forwarded previously.

\begin{tabular}{|r|r|r|r|r|}
\hline HOST & $\begin{array}{l}\text { Signal } \\
\text { Strength }\end{array}$ & Last & Clicks & Set \\
\hline $\mathrm{C}$ & $\mathrm{S}$ & 10.33 & 7 & SC \\
\hline $\mathrm{G}$ & $\mathrm{W}$ & 10.26 & 5 & WC \\
\hline
\end{tabular}

Fig 5.1.3 Signal Stability table for SSA

The propagation stops when the destination is reached or the node having a non stable route to the destination is reached, on which event a ROUTE_REPLY packet is sent.

The ROUTE_REPLY packet should travel in the reverse direction of the ROUTE_REPLY.

On its way back, each intermediate node can set up the next hop leading to the destination in its routing table. This is because SSA takes the next hop routing approach. Besides there are some "gratuitous" routes that can be added to the route table during the transmission of the "ROUTE REPLY" packet.

If multiple ROUTE_REPLY are received by the source, it can choose the one with the best quality to use. If the source fails to receive a ROUTE_REPLY packet after a timeout period, it can broadcast another ROUTE_REQUEST with other quality options to find a weaker route.

5.1.4 Route Discovery of AODV- the AODV routing protocol [13] is based on the DSDV protocol. It approves the DSDV by using an on demand philosophy to reduce the route maintenance costs, so hosts that are not on active path do not have to maintain or exchange any control information. Each host maintains its own destination sequence like DSDV to prevent looping and compare the freshness between routes.

A host broadcasts a ROUTE_REQUEST packet to its neighbors when it determines that it needs a route to destination but does not have one available. If a neighbor is an intermediate host and doesn't have any route to destination it rebroadcasts the ROUTE_REQUEST packet. If the neighbor is a destination host or an intermediate host with the route of the destination number no less than that in the route request packet. The neighbor can reply to the request of the source host by using a ROUTE_REPLY packet containing its own destination sequence number, following reverse link leading to the source. On the ROUTE_REPLY's way back to the source, the next hop routing entry can be created in each intermediate host's routing table.

5.1.5 Route Discovery of TORA- the temporary ordered routing algorithm (TORA) is characterized by a multi path routing capability [12]. Each mobile host is associated with a height metric. A wireless link is then assigned a direction by going from the host with a higher metric to the one with a lower metric. By doing so, the network can be regarded as a DAG (directed acyclic graph) with the destination host as the sink. In graph theory, a sink is a node in a directed graph with no outgoing links.

The formation of a DAG is done by broadcasting a query from the source host towards the destination host, similar to either protocol. To send the data packet, a host simply forwards the packet to any neighboring host with a lower metric. Any host receiving the data packet will do the same thing. Since the network is maintained as DAG the data packet will do the same thing. Since the network is maintained as a DAG, the data packet will eventually reach the destination. With such multi path property, one may balance / distribute traffic by a randomized technique. Also, some level of fault tolerance to route breakage can be provided.

In the route discovery algorithm given above DSR and SSA search the optimal routes randomly among the available node, while AODV and TORA algorithm search for routes on the basis of broadcasting in the neighbors which in turn again broadcasts to its neighbors. Hence in both the cases the search space with respect to each node is vast i.e. all the nodes present in the network.

The thesis proposes an algorithm which aims at acquiring the same optimal route but by reducing the search space from random to a confined area within a certain threshold which is decided basing on certain metric of calculation.

\section{Proposed Algorithm - for Optimal Route Discovery}

In ACO implemented Routing Protocol the traditional tables are replaced with the pheromone tables. These tables maintain the distance of all the nodes from each node which is calculated on the basis of Round trip time of the hello message. The pheromone value can be made inversely proportional to the RTT values, where highest RTT value has the lowest pheromone associated to it. From these distance the threshold value $(\delta)$ is calculated which depends on the battery status of the node. The node within the threshold forms the 1 hop neighbor. The node then forms clusters which comprise of those nodes which are within the threshold taking into consideration that none of clusters should be completely disjoined from all other clusters. Once the cluster www.iosrjournals.org 
is formed the nodes now generates ants with memory transmiting it to only those nodes that lies within 1 hop range. These ants carry with them list of nodes that are the one hop neighbor of the source node along with its RTT values. using these messages the node now calculates different routes to reach different nodes within its network.

From these different routes the optimal route is now calculated which has the highest pheromone value.

After the optimal tables have been made by all the nodes, for all the nodes within its cluster the tables are exchanged and finally this table is used to calculate the optimal route from each node to each other node within its network. The reduction of search space of every node to search for other nodes reduces

1. Distance that needs to be covered by a message generated by a node thus reducing the battery power consumption since power consumption is directly proportional to the distance traveled by message.

2. Routing overhead ratio since the no of nodes the message is to be transmited per node reduces.

Routing Overhead Ratio is defined as the "no of Routing Packet transmitted per data packet delivered at the destination i.e each hopwise transmission of routing packet considered as 1 transmission .

Hence considering the routes already available with a node it can calculate the optimal route to reach the destination within its network range.

The above idea is summed up into algorithm as follows:

\subsubsection{Algorithm for Optimal Route Discover -}

step 1: Using round trip time calculate the distance of all nodes present within the network.

Step 2: find the average of the Round Trip time value to determine the threshold.

Step 3. Using K-Means clustering cluster the nodes within the threshold values.

Step 4. Each node sends message to all other nodes within the cluster.

Step 5. Tables of the nodes for each other node present in the cluster is obtained.

Step 6. Tables are exchanged among the nodes.

Step 7 After exchanging the table use the table for finding the optimum route to each node present within the network with the help of the RTT values.

Step 8 Calculate the hop count from every node to all other nodes in the network

Algorithm for calc
Step 1: check the status of the battery power.

Step 2: if battery status is greater than half of the battery power capacity

Step 3: calculate the average of all the RTS values

Step 4: $\quad$ else

If battery power is more than battery capacity

Step 5: else

Threshold $(\delta)$ is the lowest RTS value or second lowest RTS value

Step 6: return $(\delta)$

Step 1: for $\mathrm{i}=1$ to nodes present

Algorithm to form clusters for node -

Step 2: for $\mathrm{j}=1$ to no of nodes present

Step 3: if (distance between the nodes $\mathrm{A}$ and $\mathrm{I}<\delta$ )

Step 4: end

Cluster $[\mathrm{i}, \mathrm{j}]=$

Example for the above algorithm

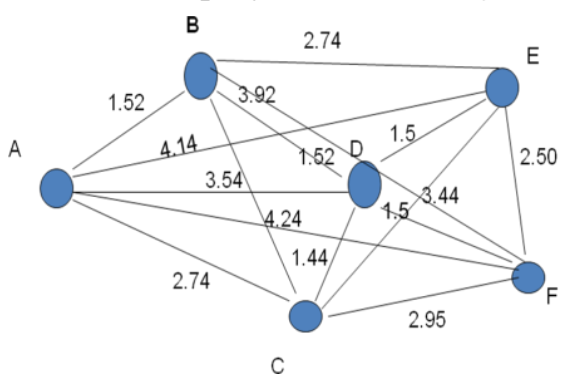




\begin{tabular}{|c|c|c|c|c|c|c|}
\hline & A & B & C & D & E & F \\
\hline A & X & 1.52 & 2.74 & 3.54 & 4.14 & 4.24 \\
\hline B & 1.52 & X & 3.54 & 1.52 & 4.14 & 4.24 \\
\hline C & 2.74 & 4.24 & X & 1.44 & 3.44 & 2.95 \\
\hline D & 3.54 & 1.52 & 1.44 & X & 1.5 & 1.5 \\
\hline C & 4.14 & 2.74 & 3.44 & 1.5 & X & 2.5 \\
\hline E & 4.24 & 3.92 & 2.92 & 1.5 & 2.5 & X \\
\hline
\end{tabular}

TABLE FOR NODE A

\begin{tabular}{|l|l|}
\hline B & $\mathbf{1 . 5 2}$ \\
\hline C & $\mathbf{2 . 7 4}$ \\
\hline D & 3.54 \\
\hline E & 4.14 \\
\hline F & 4.24 \\
\hline
\end{tabular}

The threshold value for the above table is 3.236 .

Similarly calculating the threshold values for all the nodes gives the value

\begin{tabular}{|l|l|}
\hline A & 3.236 \\
\hline B & 2.992 \\
\hline C & 2.962 \\
\hline D & 1.9 \\
\hline E & $\mathbf{2 . 8 6 4}$ \\
\hline F & $\mathbf{3 . 0 2 2}$ \\
\hline
\end{tabular}

The above given threshold values give us the range of radius within which gives us the range of radius within which our immediate neighbors lies. The nodes within the threshold are the neighbors that come within the one-hop range of the node. This threshold is based upon the average values of all the RTS if the battery power is more than half of the total battery capacity. Else it takes the lowest distance of RTS as its threshold $(\delta)$ value thus saving the battery power.

Formation of the cluster: the formation of cluster is done such that no cluster is completely disjoined from the rest of the clusters.

Finding the optimum path from node A to all other nodes in the network

\begin{tabular}{|c|c|}
\hline $\begin{array}{c}\mathrm{B} \\
\text { Direct route } \\
\end{array}$ & $\mathrm{A} \rightarrow \mathrm{B}: 1$ hop $\mathrm{RTT}$ value $=1.74$ \\
\hline $\begin{array}{c}\mathrm{C} \\
\text { Direct route }\end{array}$ & $\mathrm{A} \rightarrow \mathrm{C}: 1$ Hop $\mathrm{RTT}$ value $=2.74$ \\
\hline $\begin{array}{c}\mathrm{D} \\
\text { No direct routes, } 2 \text { hops }\end{array}$ & $\begin{array}{l}\mathrm{A} \rightarrow \mathrm{B} \rightarrow \mathrm{D} \\
\mathrm{A} \rightarrow \mathrm{C} \rightarrow \mathrm{D} \text { T value }=3.04 \\
\mathrm{RTT} \text { value }=4.18\end{array}$ \\
\hline $\begin{array}{c}\mathrm{E} \\
\text { No direct routes, } 3 \text { hops }\end{array}$ & $\begin{array}{ll}\mathrm{A} \rightarrow \mathrm{B} \rightarrow \mathrm{D} \rightarrow \mathrm{E} & \mathrm{RTT} \text { value }=4.54 \\
\mathrm{~A} \rightarrow \mathrm{C} \rightarrow \mathrm{D} \rightarrow \mathrm{E} & \mathrm{RTT} \text { value }=5.68\end{array}$ \\
\hline \begin{tabular}{l}
\multicolumn{1}{c}{ } \\
No direct routes , 3 \\
hops
\end{tabular} & $\begin{array}{l}\mathrm{A} \rightarrow \mathrm{B} \rightarrow \mathrm{D} \rightarrow \mathrm{F} \\
\mathrm{A} \rightarrow \mathrm{C} \rightarrow \mathrm{D} \rightarrow \mathrm{F}\end{array}$ \\
\hline
\end{tabular}




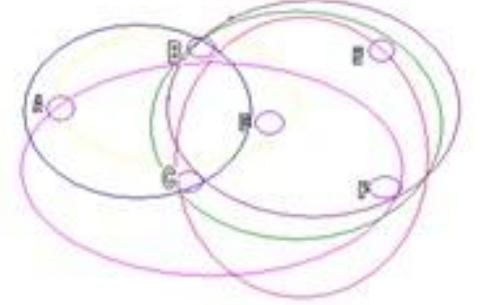

The above example shows the way in which the optimum route from each node to each other node is calculated with the help of a single hop.

Routing overhead ratio: The routing overhead ratio calculated using the above definition i.e packets transmited per packets received where each hopwise transmission of routing packets is considered as 1 transmission,for the route search where a node ony uses its next hop and accordingly transmits messages in case of no link failure or ideal scenario ,for 1 hop neighbor can be calculated as .

Packet transmited from source to destination $=1$

Packet delivered at destination $=1$

Routing overhead ratio is $1 / 1$

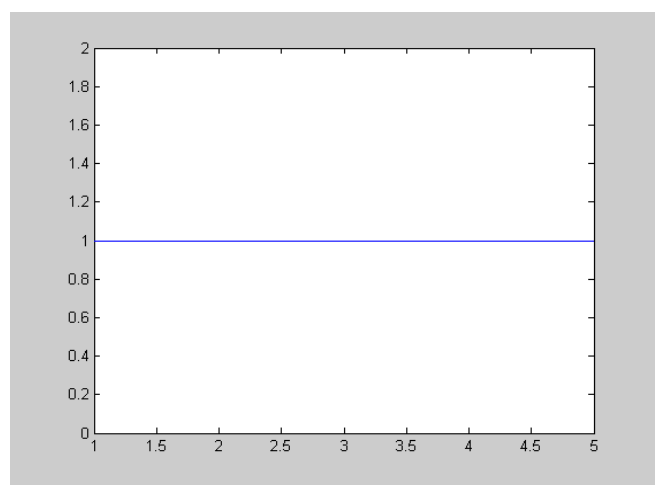

Where as for the techniques with the random search space even for 1 hop neighbour gives a value $=2 / 1$ $, 3 / 1,4 / 1$ which gives the graph of the following forms:

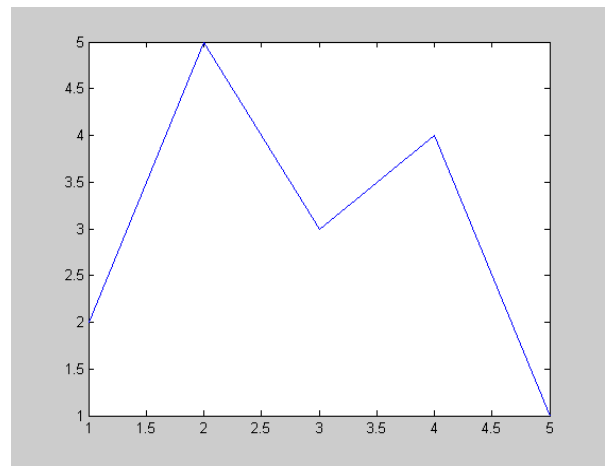




\section{STATE FLOW DIAGRAM}

\section{Random Search:}

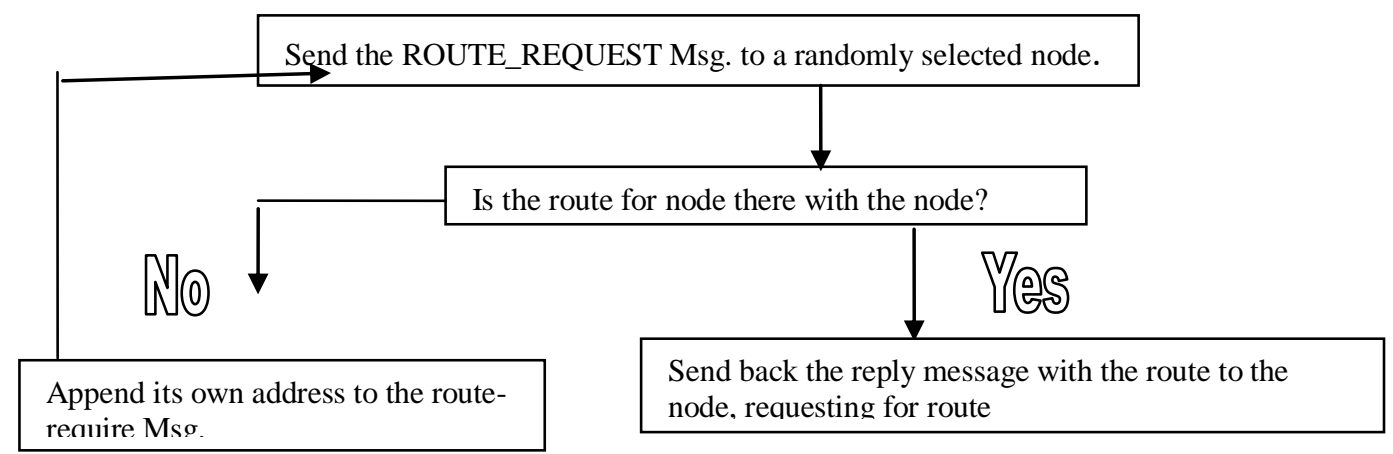

\section{Proposed Work: -}

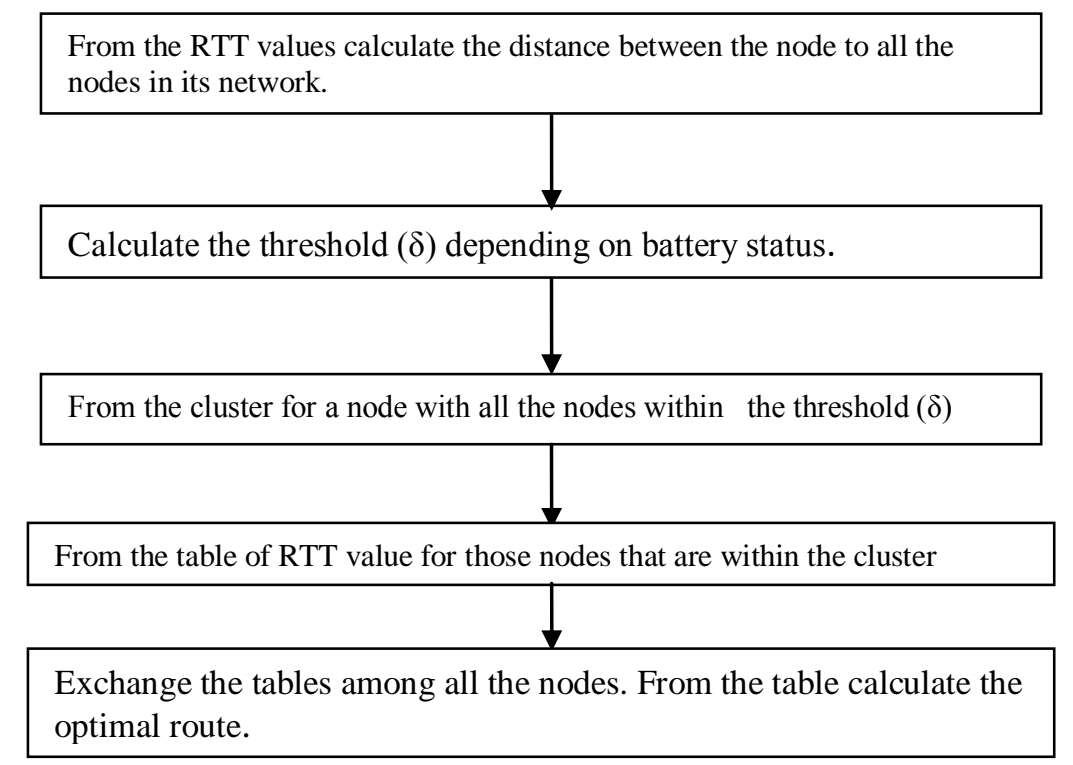

VIII. Conclusion

The above algorithm aims at proposing a technique, which reduces the routing overhead ratio as well as the battery consumption, which are two major resources. The ad-hoc network is generally needed in places affected by natural calamity or in cases of defence services where theses resources are scarcely available. There are even conditions where for days together the battery cannot be replaced or where the communication means required is fast reliable and optimal.

The algorithm hence aims at conserving the power of the battery by relating it with the calculation of the threshold value, which acts as the deciding factor of immediate neighbouring node and thereby reduces the search space for the node for searching the optimal route for a node to other nodes. The searching of the nodes is done by multicasting the ROUTE_REQUEST packet instead of broadcasting technique thereby reducing the search space due to which the routing overhead ratio is also reduced for the route searching which in turn reduces the bandwidth consumption and the random search for the route is confined to the neighbouring node to each of the node within threshold. After acquiring the route to each of the node the tables are exchanged and the different routes to all the nodes in the network is acquired. 
The optimal route is calculated on the basis of RTT values calculated through hello message.

The optimal route is also guided by other factors like

1. Signal stability strength

2. Message Overhead

3. Attenuation

4. Signal fading

5. Signal Overlapping etc.

These values are calculated by the artificial ants, generated by the node in the ant colony implementation on the ad-hoc network that are sent on the routes calculated above.

Taking into account the above factors the probability for selecting next hop for a node to other node is calculated basing on the priority and the requirement for the route. These routes in the tables are now associated with the pheromone amount deposited where the pheromone amount associated with the routes is basing upon their quality of services. The best route available is thus associated with the highest pheromone count.

\section{Future work -}

The above given algorithm is more suitable for a network with few nodes or scarcely populated nodes. With scalability or huge networks with large no of nodes lot of memory is used in storing the tables and exchanging of those tables. In future these factors can be focused while searching for optimal routes .

\section{References}

[1] Jan Komorowski, Zdzislaw Pawlak, L. P. A. S. Rough sets: a tutorial, 1998.

[2]. Pawlak, Z. Rough sets: Theoretical aspects of reasoning about data. Kluwer Dordrecht,1991.[

[3]. Adrzej Skowron, J. S. Tolerance approximation spaces. Fundamental Informaticae 27, 2-3 (1996), 245 -253.

[4] Saori Kawasaki, Ngoc Binh Nguyen, T. B. H. Hierarchical document clustering based on tolerance rough set model. In Principles of Data Mining and Knowledge Discovery, 4th European Conference, PKDD 2000, Lyon, France, September 13-16, 2000,Proceedings (2000), D. A. Zighed, H. J. Komorowski, and J. M. Zytkow, Eds., vol. 1910 of Lecture Notes in Computer Science, Springer.

[5] Tu Bao Ho, N. B. N. Nonhierarchical document clustering based on a tolerance rough set model. International Journal of Intelligent Systems 17, 2 (2002),199\{212.

[6]. Jiawei Han, M. K. Data Mining: Concepts and Techniques, 1st ed. Morgan Kaufmann,2000.

[7] Porter, M. F. An algorithm for su $\pm x$ stripping. In Readings in Information Retrieval, P. W. Karen Sparck Jones, Ed. Morgan Kaufmann, San Francisco, 1997, pp. $130\{137$.

[8]. Weiss, D. A clustering interface for web search results in polish and english, 2001.

[9]. Osinski, S. An algorithm for clustering of web search result. Master's thesis, Poznan University of Technology, Poland, June 2003.

[10] Zamir, O., and Etzioni, O. Grouper: a dynamic clustering interface to web search results. Computer Networks (Amsterdam, Netherlands: 1999) 31, 11-16 (1999), 1361-1374.

[11]. Smadja, F. A. From n-grams to collocations: An evaluation of xtract. In 29th Annual Meeting of the Association for omputational Linguistics, 18-21 June 1991, University of California, Berkeley, California, USA, Proceedings (1991),

[12]. D. Terzopoulos, X. Tu, and R. Grzeszczuk, Artificial fishes with autonomous locomotion, perception, behavior, and learning in a simulated physical world," in Artificial life 1, p. 327 ,MIY Press, 1994

[13]. K. Sims, Evolving $3^{\text {rd }}$ morphology and behavior by competition, “ in Artificial Life III, p.353, MIT Press, 1994.

[14]. Infoseek. http://infoseek.com. 